4.

\title{
Zur Frage der Hantresorption.
}

\author{
Von Dr. R. Fleischer in Erlangen.
}

In seiner Arbeit über den Zusammenhang von Hautresorption und Albuminurie 1.7. Band, 1. Heft dieses Archivs) referírt Lassar über eine grössere Beihe von Experimenten, die er an Kaninchen angestellt hat, um den Einfluss der äusseren Application verschiedener Substanzen auf den Thierkörper kennen zn lernen. Er fand dabei, dass nach Einpinselungen von Olivenöl, Räböl, Leberthran, LeinöI, Petroleum eine Infiltration der Organe des Kaninchens mit diesen Stoffen stattfindet und somit angenommen werden müsse, dass dieselben ohne Hinderniss die in tacten Hautdecken passiren und in den Kreislauf gelangen. Nach seiner Ansicht ist es schwierig, den Weg zu erkennen, welchen diese Oele durch die Haut genommen haben, jedoch sprächen Osmiumpräparate am meisten dafür, dass es die Haarfollikel seien, welche eine bequeme Eingangspforte darstellen. Nach einer längèren Erörterung der Einwirkung, welche einzelne dieser resorbirten Stoffe auf die Nieren ausüben können, wendet sich L. der Hautresorption im Allgemeinen zu und findet es auffallend, dass ich in meiner Arbeit über das Resorptionsvermögen der menschlichen Haut ${ }^{1}$ ) eine Aufnahme von Wasser, darin gelöster Substanzen und in Salbenform angewandter Medicamente geläugnet habe.

Diese meine Behauptungen basiren auf zahlreichen, mit allen möglichen Cautelen ausgeführten, zom Theil sehr mühevollen Untersuchungen am lebenden Menschen und stimmen mit den Resultaten vieler anderer Forscher überein. Man sollte nun von vornherein annehmen, dass $L$., welcher durch jene am Kaninchen gemachten Beobachtungen für das Resorptionsvermögen der Haut eingenommen war, meine Ansicht durch exactere am Menschen angestellte Experimente zu entkräften suchen würde. Nichts weniger als das. L. behilft sich mit einem allgemein gehaltenen Raisonnement. Er kann sich nicht denken, "dass die Theerintoxicationen bei äusserlicher Application dieses Mittels auf die Haut oder die Resorptionssymptome nach ausgedehnter Carbolöleinreibung allein der Aufnahme durch die Respirationsorgane zuzuschreiben seien und meint, dass die zahllosen 0effnungen, mittelst derer das Körperinnere durch die Haut mit der Aussenwelt communicirt, dieselbe zar Aufoahme flüssiger Substanzen im hohen Grade geeignet machen. Da ihm dies zur Begründung seiner Ansicht offenbar noch nicht ganz hinreichend erscheint, so führt er noch Beobachtungen von Unna an, welche beweisen sollen, dass die Haut des Kaninchens bezüglich des Resorptionsvermögens sich genau ebenso verhält wie die des Menschen. Belkanntlich hat $\mathrm{Unn}_{\mathrm{n}}$ in seiner Mittheilung üher Albuminurie während der Styraxeinreibungen Krätziger ${ }^{2}$ ) hervorgehoben, dass in Folge dieser Kur bei einer kleinen Anzahl von Fällen Eiweiss im Harn auftritt und diese tran-

1) Habilitationsschrift. Erlangen 1877. (Besold.)

$\left.{ }^{2}\right)$ Dieses Archiv 74. Band 3. Heft. 
sitorische Albuminurie jedenfalls auf die Einwirkung des durch die Haut aufgenommenen und im Harn erscheinenden Styrax auf die Capillarwände in den Nieren zu beziehen ist. Natürlich ist es $\mathrm{Unna}$, der sich viel mit Hautaffectionen beschäftigt hat, nicht eingefallen, diese Erfahrung für die Resorptionsfähigkeit intacter menschlicher Haut za verwerthen. Jedermann, der in seinem Leben Krätze bebandelt hat, kennt die dabei stets vorkommenden Kratzexcoriationen und als Complication das Eczema (daher der Name Krätzeczem). Zum Ueberfluss findet sich auch noch in den beigegebenen Krankheitsprotocollen von Un a eine eczematöse Hauterkrankung geringeren oder stärkeren Grades bei den angeführten Kranken verzeichnet. L assar setzt sich darüber hinweg; ihm erscheint die scabiöse eczematöse Haut der normalen gleichwerthig. Seinen eignen Worten nach begegnen die angeführten Beobachtungen von Unna, dass der eingeriebene Styrax im Harn wieder erscheint, von vornherein dem Einwand, dass in Beziehung auf die Durchgängigkeit der Haut ein grundsätzlicher Unterschied zwischen Mensch und Thier besteht. “

Die Unzulässigkeit solcher Schlnssfolgerungen liegt so auf der Hand, dass wir darauf verzichten, sie noch näher zu beleuchten. Dagegen wollen wir L. gern zugeben, dass die längst bekannte Resorption von Wunden aus durch Unna's Beobachtungen wieder auf's Neue bestätigt ist. Was ferner seine oben betonte Theerjntoxication betrifft, welche für eine Aufnahme durch die intacte Haut sprechen soll, so werden meines Wissens keine Theereinreibungen der normalen Haut gemacht und so beweist diese Thatsache für L. nichts. Erscheint somit die ganze Anschauung von L. als hinfällig, so bieten auch seine weiteren Auseinandersetzungen noch manche Punkte, die gegen eine strengere Kritik nicht Stich halten. L. findet die Ursache, weshalb die Resorptionsversuche der verschiedenen Arbeiten so verschiedene Ergebnisse lieferten, in der Natur des auf die Haut gebrachten Stoffs.

Da die verhornte Epidermis keine Durchlässigkeit besitze, so können nur die Ausführungsgänge der Schweiss- und Talgdrüsen, die mit öligem Secret und Fettkörnchen gefüllt sind, wodurch das Eindringen von Wasser verhindert wird, die Communication vermitteln: Dadurch erklären sich die negativen Resultate bei Versuchen mit Wasser und darin gelösten Stoffen. Dagegen stehe der Aufsaugung von solchen Stoffen nichts entgegen, welche wie das 0el sich mit dem Inhalt der Drüsensubstanz mischen, oder wie alkoholische Lösungen dieselben verfüssigen können. Es beschränke sich also die Resorption, wie dies übrigens schon von anderen Forschern oft hervorgehoben ist, auf solche Substanzen, und die darin gelösten oder fein suspendirten Ingredienzien, für die das Vorbandensein einer Fettschicht in den Ausführungsgängen der Hautdrüsen kein Hinderniss des Vordringens abgiebt. - So calculirt Lassar. - Da wir aber sehr viele Stoffe pulvern und mit 0el oder Fett fein verreiben können, welche nun in dieser Form aufgenommen werden sollen, so wäre die Resorptionsfähigkeit der intacten menschlichen Haut nach L. eine naheztr unbegrenzte. Wäre dies richtig, so hätte ich doch bei den ausgedehnten Einreibungen meiner äusseren Haut mit Veratrin und Morphinsalben $(0,5 !$ p. d. Veratrin, 1,0 Morph. acet.!) irgend einen Einfluss auf mein Allgemeinbefinden verspüren müssen. Dỉese Resultate ignorirt L. ebenso wie die anderen zahlreichen Versuche, die mit Jodkalisalben [1) Jodkali einfach mit Fett verrieben, 2) mit dem Ungt. kal. jod. 
off, 3) mit in Alkohol gelöstem Jodkali und Fettzusatz, 13 Versuche] von mir såmmtlich mit negativem Erfolg angestellt sind. Niemals sah ich auch nur Spuren von Jod im Harn anftreten, welches, wie ich vorher constatirt hatte, bei innerlicher Darreichung von 0,001! (also einer bereits für irgend einen Heileffect irrelevanten Menge) im Harn nachweisbar ist ${ }^{\mathbf{t}}$ ).

Wenn L. wieder auf jene von älteren Aerzten empfohlenen Fett- und Leberthraneinrelbungen zurückkommt, um auf diesem angewöhnlichen Wege Nährmaterial zuzuführen, so halten wir es für unnöthig, ihm darauf ernstlich zu antworten. Schliesslich bringt $\mathrm{L}$, noch eine kleine therapeutische Vorsichtsmaassregel mit seiner Annahme in Zusammenhang. Nach ihm verhütet man bei Anstellung von Sectionen mit grosser Sícherheit das Auftreten von Leichenfurunkeln und Phlegmonen, wenn man vor der Obduction die Hände und Unterarme mit 1-2 pCt. Carbolöl einreibt. Dies beruhe darauf, dass das Leichengift, welches sonst in die Mündung der Haarfollikel oder Schweissdrüsen eindringt, dieselben mit desinficirendem Oel angefüllt findet, welches eine weitere Resorption hindert und das Gift seiner entzündungserregenden Wirkung beraubt. Verstehe ich Herrn Lassar recht, so müssen Fälle von Leichenvergiftung von der intacten Haut aus sehr häufig sein. Solche Fälle sind aber meines Wissens nicht bekannt. Bei den eindeutigen Beobachtungen waren stets Riss- oder Schnittwunden oder andere Verletzungen der Haut die Infectionsorte. Wäre dagegen die Auseinandersetzung von L. zutreffend, so wäre die Leicheninfection ohne weitere Wunde ein sehr häufiges Vorkommniss und der pathologische Anatom, der Lassar's Vorsichtsmaassregel verabsäumt, würde seines Lebens nicht froh.

So lange L. nichts Besseres für seine Ansicht vorbringt, müssen wir uns seinen Aeusserungen gegenüber ablehnend verhalten. Wir sind nach whe vor der Meinang, dass von der unversehrten menschlichen Haut eine Aufsaugung von flüssigen und festen Stoffen (welche nicht corrodirend wirken) nicht stattfindet. Für uns bleibt der Nachweis oder Nichtnachweis der angewandten Stoffe im Harn und Speichel und der Einfluss auf das Allgemeinbefinden maassgebend. Wollten wir auch eine Resorption von so minimalen Mengen annehmen, dass sie durch unsere Reactionen nicht mehr erkannt werden, so könnten wir denselben keinen Heileffect zusprechen, da es sich dann um Quantitäten handelt, welche bei einer rationellen Behandlung der ganzen Frage nicht in Betracht kommen können.

1) Ich habe kürzlich noch einen Versuch gemacht und die Resorptionsbedingungen im Lassar'schen Sinn möglichst gủnstig za gestalten gesucht. $1 \mathrm{Grm}$. Jodkall wurde in Glycerin gelöst und nach Mischung mit olivenöl in dieser Linimentform in die Unterextremität einer Kranken eingerieben und dann von der äussern Haut möglichst gut abgeschlossen. Der Harn der folgenden 48 Stunden (während derer die Salbe auf der Haut blieb) enthielt k'eine Spur von Jod. 